\title{
Novel Method to Improve the Uniformity of 7T Body MR Images
}

\author{
Bu S. Park (iD ${ }^{1}$ and Sunder S. Rajan ${ }^{2}$ \\ ${ }^{1}$ Division of Cellular and Gene Therapies (DCGT)/OTAT/CBER, Food and Drug Administration (FDA), Silver Spring, \\ MD 20993-0002, USA \\ ${ }^{2}$ Division of Biomedical Physics (DBP)/OSEL/CDRH, Food and Drug Administration (FDA), Silver Spring, MD 20993-0002, USA \\ Correspondence should be addressed to Bu S. Park; bu.park@fda.hhs.gov
}

Received 9 November 2020; Revised 30 March 2021; Accepted 7 April 2021; Published 6 May 2021

Academic Editor: Zhong Chen

Copyright (C) 2021 Bu S. Park and Sunder S. Rajan. This is an open access article distributed under the Creative Commons Attribution License, which permits unrestricted use, distribution, and reproduction in any medium, provided the original work is properly cited.

\begin{abstract}
When using ultrahigh-field MR systems (7T), the variations in the RF magnetic field can lead to significant loss in image uniformity. To optimize the overall MR image quality, the image region is divided into multiple smaller regions of interest (the ROIs), which can be independently optimized using transmit array optimization techniques including RF shimming, to improve RF magnetic fields and image intensity. Electromagnetic numerical simulations and corresponding transverse magnetization (| $\mathrm{M}_{\mathrm{t}} \mid$ ) acquired using the Bloch equation-based MRI simulator are used to evaluate the proposed method. Compared to the simulation results of quadrature driving method, mean and standard deviation (SD) of $\left|M_{\mathrm{t}}\right|$ in the full image (an inner diameter of $500 \mathrm{~mm}$ ) were improved $47 \%$ (mean) and $48 \%(\mathrm{SD})$, whereas $94 \%(\max )$ and $97 \%$ (mean) improved in the unaveraged SAR using the proposed method. The uniformity of $\left|M_{\mathrm{t}}\right|$ acquired using the method was especially improved in the peripheral region of the selected phantom image compared to that of other methods. The proposed method using multiple independently optimized ROIs and numerical simulations significantly improved the uniformity of $\left|M_{\mathrm{t}}\right|$ body images at 7T. This technique would be generally applicable to any high-field strength MR systems, which generate short RF wavelengths compared to the field of view.
\end{abstract}

\section{Introduction}

To acquire magnetic resonance (MR) images of high resolution and increased signal-to-noise ratio (SNR), a higher static magnetic field $\left(\left|B_{0}\right|\right)$ is needed [1-6]. This is because the intrinsic SNR (ISNR) in magnetic resonance imaging (MRI) is proportional to the square of $\left|B_{0}\right|\left(\operatorname{ISNR} \propto\left|B_{0}\right|^{2}\right)[1,2]$. However, as $\left|B_{0}\right|$ is increased, the absorbed power increases significantly $[5,7]$, and magnetic field inhomogeneity caused by a wavelength effect results in decreased image intensity uniformity [6-8]. These effects are significant in the imaging of human tissue using 7T MRI systems [6], the highest field approved for MRI by the FDA. Many different methods have been studied to solve the issues of image uniformity and absorbed power of high-field MRIs. Some of the examples are multichannel excitation using a shielded birdcage coil $[9,10]$, transmit array with radiofrequency (RF) shimming [11-15], composite pulse [16, 17], spoke pulse [18-20], different transmit RF coil designs [21-24], high dielectric constant
(HDC) materials [5, 7, 25-28], and simultaneous transmit excitation of phased array and volume coil [8]. Specifically, Taracila [9] showed image uniformity was improved by applying a series of multiple current distributions on the entire birdcage coil using different modes of the birdcage coil and then combining individually acquired images at 300-400 MHz. Orzada et al. [10] showed similar results using time-interleaved acquisition of modes (TIAMO) methods. Collins et al. [11] showed that optimization of amplitude and phase of current at each transmit channel; i.e., RF shimming could significantly improve flip angle uniformity for highfield human head MRIs with operating frequencies between $300 \mathrm{MHz}$ and $600 \mathrm{MHz}$. Setsompop et al. [18] showed that custom designed spatially selective RF waveforms, called spoke pulses, with an 8-channel transmit array could improve image uniformity compared to the standard slice selective method for 3.0 T human head MRI. Erturk et al. [21] designed a 16-channel transmit and receive array, consisting of eight combined loop-dipole elements, which improved 
SNR, peak rotating RF transmit magnetic field amplitude (| $\mathrm{B}_{1}^{+} \mid$), and specific absorption rate (SAR) compared to imaging with the 16-channel microstrip line and 10-channel fractionated dipole antenna design. Yang et al. [25] showed improved SNR from brain and reduced SAR with HDC material of water (conductivity $(\sigma)=0.0047 \mathrm{~S} / \mathrm{m}$ and relative permittivity $\left.\left(\varepsilon_{\mathrm{r}}\right)=78\right)$ for $3 \mathrm{~T}$ head MRI. Some researchers used the above high-field RF optimization methods for imaging of specific regions of interest (ROIs) such as prostate [29], liver [30], and head imaging [31, 32]. However, most of the previous methods have limitations on making uniform RF magnetic fields and image intensity when the size of a imaging region was large enough to over one wavelength $(\lambda)$ and $\left|B_{0}\right|$ is $>3 \mathrm{~T}$; e.g., image nonuniformity was evident for whole body MR images at $7 \mathrm{~T}$ or higher field strengths in the previous research [21-23].

The wavelength of electromagnetic fields would be decreased inside the human body because of an increased $\varepsilon_{r}$ value $\left(\lambda \propto 1 / \sqrt{\varepsilon_{r}}\right)$ compared to the $\lambda$ in the free space. Specifically, $\varepsilon_{r}$ of an average human muscle is 63.9 at $128 \mathrm{MHz}$ and 59.0 at $300 \mathrm{MHz}[33,34]$, whereas $\varepsilon_{r}$ is 1 in the free space at any frequency. Therefore, one wavelength of electromagnetic fields in the average human muscle is $0.29 \mathrm{~m}$ at $128 \mathrm{MHz}$ and $0.13 \mathrm{~m}$ at $300 \mathrm{MHz}$, whereas it is $2.34 \mathrm{~m}$ at $128 \mathrm{MHz}$ and $1.0 \mathrm{~m}$ at $300 \mathrm{MHz}$ in the free space. Therefore, it would be challenging to make uniform RF magnetic fields inside the human body with the decreased $\lambda$ of electromagnetic fields.

Based on the results shown in previous research studies [9-18, 21-23], the maximum size of a region that can make uniform magnetic fields and image intensity at 7.0 T would be less than or approximately one wavelength. However, the waist size of an average man is about $0.83-0.99 \mathrm{~m}$ [35] (the diameter is about $0.26-0.32 \mathrm{~m}$ with assumption of a cylinder), which is much bigger than one wavelength at 7.0 T. Therefore, it would be difficult to make uniform magnetic fields and image intensity within the whole human body including a waist at $7.0 \mathrm{~T}$ with any known methods; for example, Vaughan et al. [2] showed MR image intensity in the boundary region was decreased about $43 \%$ compared to that in the center region of a brain at $300 \mathrm{MHz}\left(\lambda 0.14 \mathrm{~m} ; \varepsilon_{r}=51.9\right.$ in the average human brain at $300 \mathrm{MHz}$ ), whereas about $23 \%$ decreased at $170 \mathrm{MHz}$ $\left(\lambda 0.23 \mathrm{~m} ; \varepsilon_{r}=58.2\right.$ in the average human brain at $\left.170 \mathrm{MHz}\right)$.

A new method is presented using RF shimming with multiple ROIs, RSMR, to improve uniformity of the RF magnetic field and corresponding MR image intensity at $7 \mathrm{~T}$. Specifically, if the ROI is larger than one wavelength, the region would be divided into multiple smaller regions. After that, independent optimization techniques including RF shimming would be applied to each region to improve RF magnetic fields and image intensity. The RSMR method was implemented using the finite difference time domain (FDTD) numerical simulations and the Bloch equation-based MRI simulator [36].

\section{Methods}

Numerical simulations and optimization were performed using 16-channel transmit array, cylindrical body phantom, whole-body human female model named Ella from the Virtual Family [37], and the MRI system simulator
[36] for body imaging. The experimental verifications for body imaging were not shown in this study because of difficulties to acquire experimental data. All FDTD numerical simulations were performed at $300 \mathrm{MHz}$ with commercially available software (xFDTD; Remcom, Inc; State College, PA, USA), and analysis of the results was performed in MATLAB (The MathWorks, Inc., Natick, MA, USA). The magnitude of transverse magnetization (| $\mathrm{M}_{\mathrm{t}} \mid$ ) and MR images of the head were acquired using experimentally acquired 8-channel transmit rotating RF magnetic field $\left(\mathrm{B}_{1}^{+}\right)$of each channel, optimization methods, and the Bloch equation-based MRI system simulator $[17,36]$.

2.1. Overall Flow Chart of the Optimization. Figure 1 shows the overall flowchart of acquiring optimized MR images using multiple ROIs considering wavelength within the sample and electromagnetic properties of the sample and transmit array optimization methods, e.g., RF shimming for this study. MR images are acquired sequentially after each ROI optimization. The image data are combined after all the ROIs have been optimized and imaged.

2.2. Computational Model of RF Coils. The 16-channel transmit array for body imaging was used in this study (Figure 2). The 16-channel transmit array has an inner diameter (ID) of $620 \mathrm{~mm}$, inner rod width of $30 \mathrm{~mm}$, outer rod width of $60 \mathrm{~mm}$ (Figure 2(b), red and white arrows), gap between inner and outer rods of $10 \mathrm{~mm}$, and length of $620 \mathrm{~mm}$ (Figures 2(a) and 2(c)). The RF shield having ID of $827 \mathrm{~mm}$ and length of $845 \mathrm{~mm}$ was used in this study (Figures 2(a) and 2(c)) [17]. A cell size of the 16-channel transmit array and RF shield for FDTD simulations was $3 \times 3 \times 3 \mathrm{~mm}^{3}$.

2.3. Computational Model of Sample and Human. A cylindrical uniform phantom used for body imaging had an ID of $540 \mathrm{~mm}$ and length of $620 \mathrm{~mm}$. The phantom was composed of a material having electrical properties of $\sigma=0.79 \mathrm{~S} / \mathrm{m}$ and $\varepsilon_{\mathrm{r}}=59.0$, which are the same as the properties of average human muscle at $300 \mathrm{MHz}[33,38]$. The optimized size of each ROI was calculated by checking | $\mathrm{Mt}$ | uniformity using the RF shimming method (Figure 3 and Table 1). Using the optimized size, the phantom was divided into three regions (regions I $(0-100 \mathrm{~mm})$, II (100-200 mm), and III $(200-260 \mathrm{~mm})$ ) for optimization (Figures 2(b) and 4, Table 2), and the matrix size for the phantom simulations was $258 \times 259 \times 250 \mathrm{~mm}^{3}$. The regions were determined based on the one wavelength size (about $130 \mathrm{~mm}$ at $300 \mathrm{MHz}$ within the average human muscle) and changed by a $10 \mathrm{~mm}$ until finding a maximum size having a good uniformity of $\left|M_{t}\right|$ (more than $90 \%$ of the best value).

The Ella model for body imaging included 37 anatomical structures, with a $3 \mathrm{~mm}$ isotropic resolution, and the electromagnetic tissue properties were assigned based on literature $[33,34,38,39]$. The model was positioned to have the 


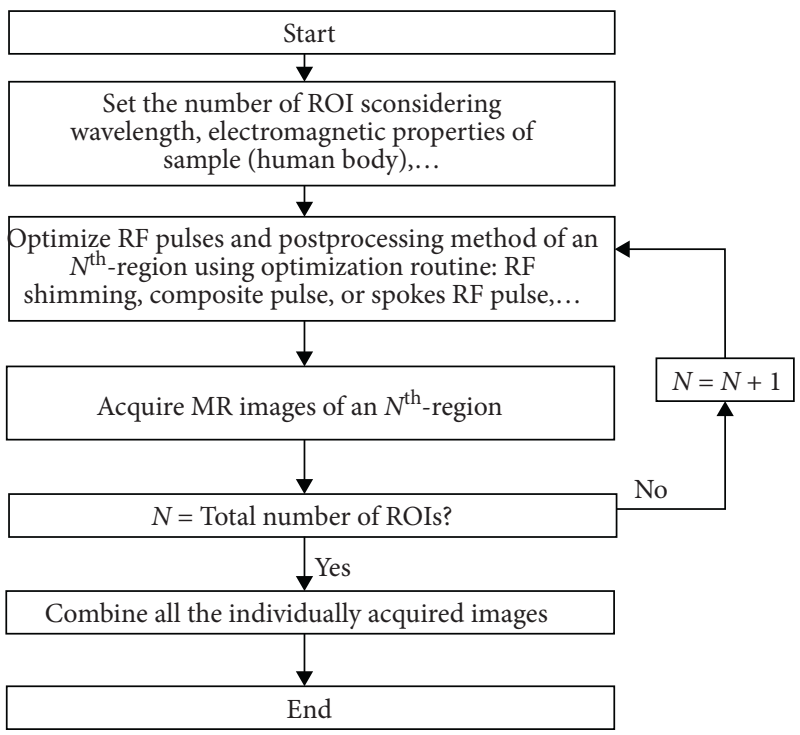

FIGURE 1: Overall flowchart of acquiring optimized MR images using multiple regions of interest (ROIs) and transmit array optimization methods, e.g., RF shimming, composite pulse, and spokes RF pulse.

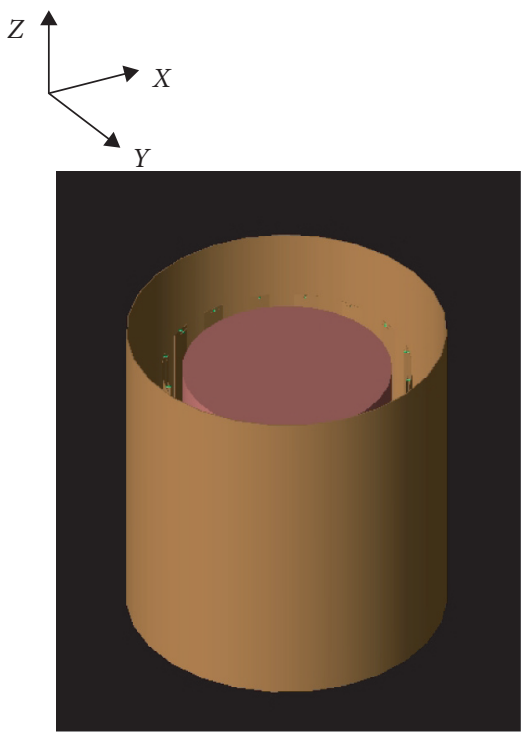

(a)

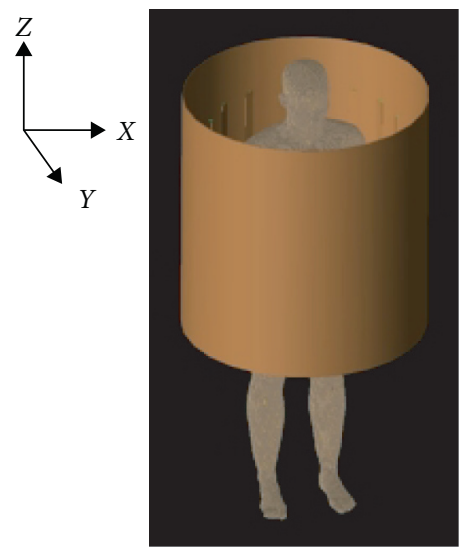

(c)

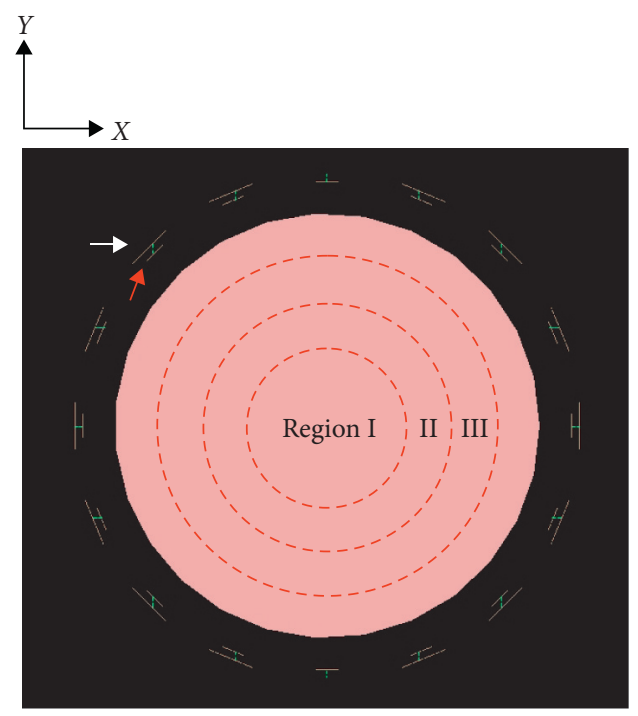

(b)

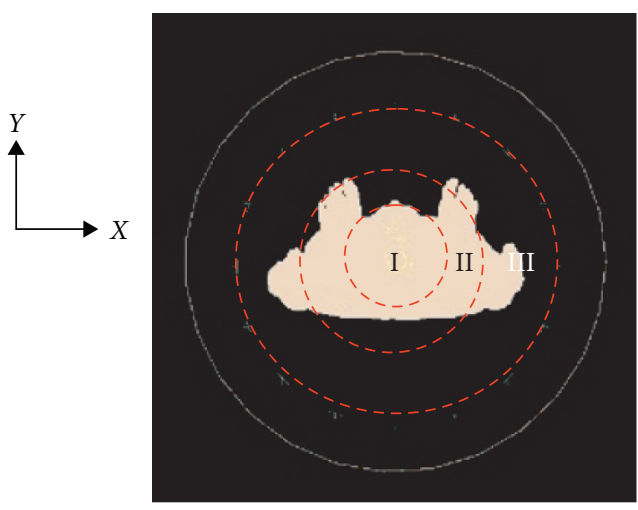

(d)

FIGURE 2: Geometrical models and multiple ROIs used in this study: (a) 16-channel transmit array (ID = $620 \mathrm{~mm}$ and length $=620 \mathrm{~mm}$ ), RF shield (ID $=827 \mathrm{~mm}$, length $=845 \mathrm{~mm}$ ), and cylindrical uniform phantom $\left(\mathrm{ID}=540 \mathrm{~mm}\right.$, length $=620 \mathrm{~mm}, \sigma=0.79 \mathrm{~S} / \mathrm{m}$, and $\varepsilon_{\mathrm{r}}=59.0($ ave . muscle at $300 \mathrm{MHz}$ )) at 7.0 T MRI; (b) multiple ROIs considering wavelength and electromagnetic properties of sample (inner rod (red arrow) width of $30 \mathrm{~mm}$ and outer rod (white arrow) width of $60 \mathrm{~mm}$ ); (c, d) with human body model of Ella. 


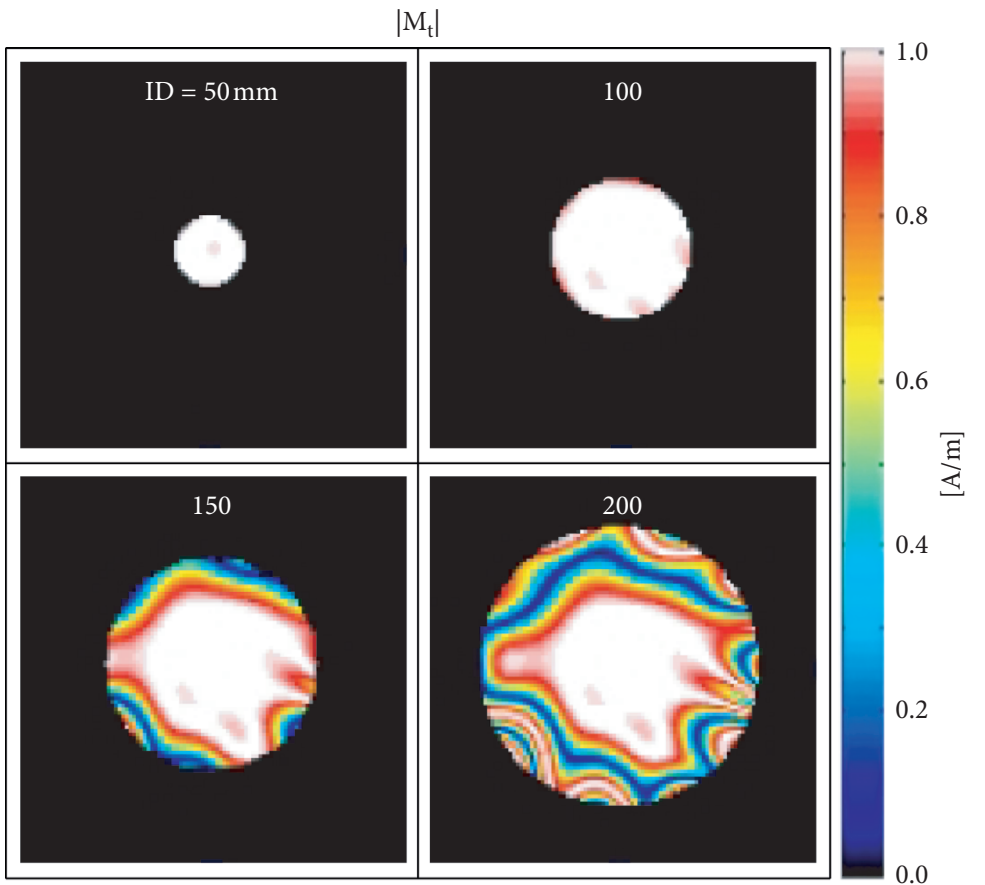

(a)
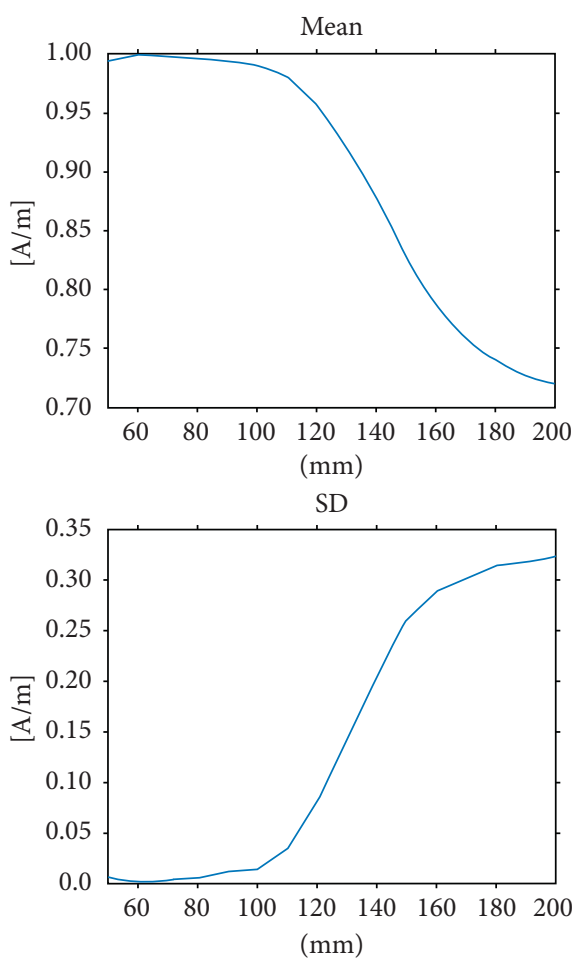

(b)

FIGURE 3: Numerical simulation results of transverse magnetization $(|\mathrm{Mt}|)$ with different inner diameters (IDs) of region of interest (ROI) and designed RF shimming method (a). Mean and standard deviation (SD) of $|\mathrm{Mt}|$ with different IDs (b).

TABLE 1: Mean and SD of 2D- $\left|M_{t}\right|$ depending on the inner diameter (ID) of the cylindrical phantom (Figures 2(a) and 3).

\begin{tabular}{lcccc}
\hline & ID $=50 \mathrm{~mm}$ & 100 & 150 & 200 \\
\hline Mean $\left|M_{t}\right|-2 \mathrm{D}(\mathrm{A} / \mathrm{m})$ & 0.994 & 0.990 & 0.828 & 0.720 \\
SD $\left|M_{t}\right|-2 \mathrm{D}$ & 0.006 & 0.013 & 0.260 & 0.304 \\
\hline
\end{tabular}

center of the heart corresponding to the center of the body transmit array in the $Z$-direction (Figure 2(c)). The Ella model was divided into two different cases: cases I and II. Case I has three different concentric regions of interest I $(0-80 \mathrm{~mm})$, II $(80-150 \mathrm{~mm})$, and III $(150-500 \mathrm{~mm})$ ) for optimization (Figure $2(\mathrm{~d})$ ), whereas case II has two regions of interest I (0-200 mm) and II (200-500 mm). Case II regions were selected to decrease the scanning time with a minimum sacrifice of $\left|\mathrm{M}_{\mathrm{t}}\right|$ uniformity (Table 3). Also, the matrix size for numerical simulations using the Ella model was $258 \times 259 \times 589 \mathrm{~mm}^{3}$. A multiresolution matrix size was used for the numerical simulations to save a computational time; i.e., $3 \times 3 \times 3 \mathrm{~mm}^{3}$ (within RF coils, sample, and human model) and $20 \times 20 \times 20 \mathrm{~mm}^{3}$ (outside of the RF coils, sample, and human model).

2.4. Computational Model of Driving Methods. Each port of the 16-channel transmit array for body imaging was excited with a voltage source having a magnitude of $1 \mathrm{~V}$ and a phase equal to the azimuthal position of the element in series with a $50-\mathrm{ohm}$ resistor connected in serial. The RF shimming technique [11-15] was used for optimization of each region in phantom and human body imaging. Specifically, the amplitude and phase of each channel were optimized to produce the most homogeneous $\left|M_{t}\right|$ and the lowest SAR within the ROI by minimizing the SD of $\left|M_{t}\right|$ and/or maximum SAR over the ROI. During optimization, a simple cost function was used to balance the uniformity of $\left|M_{t}\right|$ and SAR.

$$
\eta \times\left|M_{t}\right|_{\text {inhomogeneity }}+(1-\eta) \times \mathrm{SAR}_{\mathrm{Max}}, \quad 0 \leq \eta \leq 1 .
$$

The value of $\eta$ can be varied any number from 0 to 1 for considering the uniformity of $\left|M_{t}\right|$ and SAR; however, three numbers of 0 (only considering SAR), 0.5 (half), and 1 (only considering uniformity of $\left.\left|M_{t}\right|\right)$ are shown in this study (Figures 5 and 6).

The $\left|M_{t}\right|$ was calculated, ignoring the effect of spin-lattice relaxation time $\left(T_{1}\right)$ and spin-spin relaxation time $\left(T_{2}\right)$, using the Bloch equation and $\left|B_{1}{ }^{+}\right|$of each channel acquired from numerical FDTD simulations [36]:

$$
\frac{\mathrm{d} M}{\mathrm{~d} t}=\gamma M \times \sum_{n} B_{1 n}^{+}
$$

where $M$ represents the net magnetization vector, $\gamma$ is the gyromagnetic ratio, i.e., $42.58 \mathrm{MHz} / \mathrm{T}$ for the ${ }^{1} \mathrm{H}$, and $B_{1 n}^{+}$is the complex circularly polarized component of RF magnetic field in each unit.

The SAR was calculated using the electric field distributions and tissue properties as follows: 


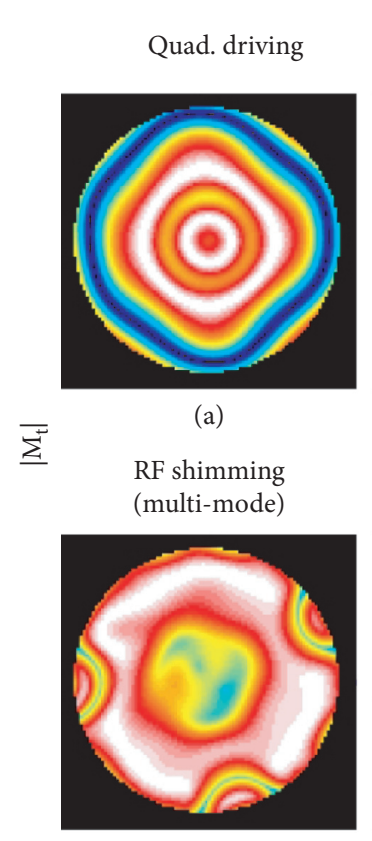

(c)

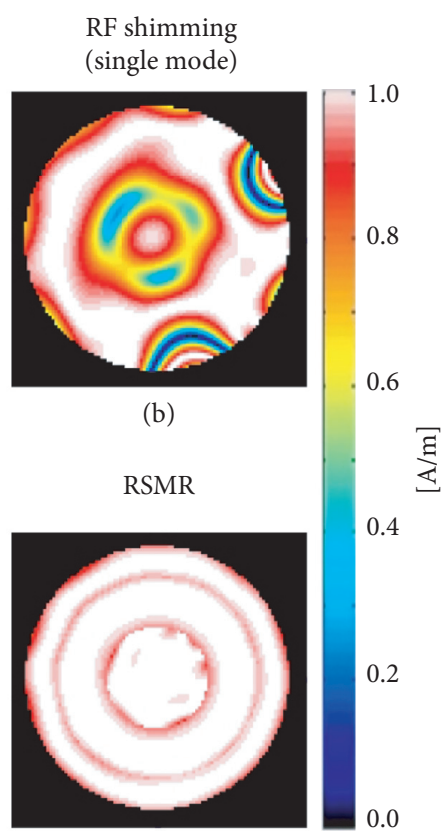

(d)

FIGURE 4: Numerical simulation results of $|\mathrm{Mt}|$ with three different methods of transmit array optimization, i.e., quadrature driving (a), RF shimming with single mode (b) and combination of multi-mode optimizations (c), and RF shimming with multiple ROIs (RSMR) (d) using the cylindrical phantom with ID of $260 \mathrm{~mm}$ at $7.0 \mathrm{~T}$.

TABLE 2: Mean and standard deviation (SD) of 2D transverse magnetization $\left(\left|M_{t}\right|-2 \mathrm{D}\right)$ within the cylindrical phantom in (Figures 2 and 4).

\begin{tabular}{|c|c|c|c|c|c|c|c|}
\hline & $\begin{array}{l}\text { Quad. } \\
\text { driving }\end{array}$ & $\begin{array}{l}\text { RF shimming } \\
\text { (single mode) }\end{array}$ & $\begin{array}{l}\text { RF shimming } \\
\text { (multi-mode) }\end{array}$ & $\begin{array}{c}\text { Region I } \\
(0-100 \mathrm{~mm})\end{array}$ & $\begin{array}{c}\text { Region II } \\
(100-200 \mathrm{~mm})\end{array}$ & $\begin{array}{c}\text { Region III } \\
(200-260 \mathrm{~mm})\end{array}$ & $\begin{array}{c}\text { RSMR } \\
\text { (combined) }\end{array}$ \\
\hline $2 \mathrm{D}(\mathrm{A} / \mathrm{m})$ & 0.61 & $0.85(39 \%)$ & $0.85(39 \%)$ & 0.99 & 0.99 & 0.98 & $0.98(61 \%)$ \\
\hline $\mathrm{SD}\left|\mathrm{M}_{\mathrm{t}}\right|-2 \mathrm{D}$ & 0.31 & $0.20(35 \%)$ & $0.15(52 \%)$ & 0.02 & 0.02 & 0.03 & $0.02(94 \%)$ \\
\hline
\end{tabular}

RSMR: RF shimming with multiple ROIs.

$$
\operatorname{SAR}=\frac{\sigma}{2 \rho} E_{x}^{2}+\frac{\sigma}{2 \rho} E_{y}^{2}+\frac{\sigma}{2 \rho} E_{z}^{2}
$$

where $E_{\mathrm{x}}, E_{\mathrm{y}}$, and $E_{\mathrm{z}}$ are the amplitudes of $x, y$, and $z$ components of electric field, $\sigma$ is the conductivity $(\mathrm{S} / \mathrm{m})$, and $\rho$ is the mass density $\left(\mathrm{kg} / \mathrm{m}^{3}\right)$.

\subsection{Single-Mode RF Shimming Vs. Multi-Mode RF Shimming.} In this study, the multi-mode RF shimming is to excite two different $B_{1}{ }^{+}$modes using static RF shimming in an interleaved acquisition based on the previous research [10] (the authors called this method as TIAMO). In this method, each result compensates each other; for example, the first image has good uniformity of $\left|M_{t}\right|$ at the center region, whereas the second one has good uniformity of $\left|M_{t}\right|$ at the boundary region. Therefore, combining the two images can improve the uniformity of $\left|M_{t}\right|$ with sacrificing increase in scanning time by a factor of two.

The single-mode RF shimming uses one $B_{1}{ }^{+}$mode; therefore, images can be acquired without increasing a scanning time.

The single-mode and the multi-mode RF shimming as well as a conventional quadrature driving method with the same transmit array, sample, and human models were used for comparison. Before optimization, all electromagnetic fields were normalized so that the dissipated power of each channel was set as $1 \mathrm{~W}$.

\section{Results}

Figure 1 shows the overall flowchart of acquiring optimized MR images using multiple ROIs considering wavelength and electromagnetic properties of sample combined with optimization methods, e.g., RF shimming [11-15], composite pulse $[16,17]$, and spoke RF pulse [18-20]. Each region of multiple ROIs was optimized individually starting with reference data and then combined into a full MR image using individually calculated $\left|\mathrm{M}_{\mathrm{t}}\right|$ of each region and $\mathrm{MR}$ simulator $[14,16,17]$. The reference data, i.e., initial values used for optimization of amplitude and phase of each channel, were selected based on the numerical simulations.

Figure 3 shows calculated $\left|\mathrm{M}_{\mathrm{t}}\right|-2 \mathrm{D}$ and corresponding mean and SD of $\left|M_{t}\right|$ (Table 1) within the cylindrical phantom using different inner diameters (IDs). Note that the mean and SD of $\left|\mathrm{M}_{\mathrm{t}}\right|$ were changed less than $10 \%$ until the ID was $100 \mathrm{~mm}$ (Table 1). The optimization of $\left|\mathrm{M}_{\mathrm{t}}\right|$ uniformity using RF shimming for each IDs was performed based on the previous research [17]. 
TABLE 3: Mean and SD of 2D- $|\mathrm{Mt}|$ and unaveraged SAR within the human body model, Ella (Figures 5 and 6).

\begin{tabular}{lcccccccc}
\hline & \multirow{2}{*}{ Quad. driving } & \multicolumn{3}{c}{ RF shimming (single mode) } & \multicolumn{3}{c}{ RSMR Case I } & \multirow{2}{*}{ RSMR Case II $(\eta=1.0)$} \\
& & $\eta=1.0$ & 0.5 & 0.0 & $\eta=1.0$ & 0.5 & 0.0 & \\
\hline Mean $\left|M_{t}\right|-2 \mathrm{D}(\mathrm{A} / \mathrm{m})$ & 0.64 & $0.89(39 \%)$ & 0.87 & 0.69 & $0.94(47 \%)$ & 0.93 & 0.51 & $0.92(44 \%)$ \\
SD $\left|M_{t}\right|-2 \mathrm{D}$ & 0.31 & $0.19(39 \%)$ & 0.20 & 0.30 & $0.16(48 \%)$ & 0.17 & 0.32 & $0.17(45 \%)$ \\
Max SAR-2D (W/kg) & 205 & $11.7(94 \%)$ & 10.2 & 6.32 & $12.3(94 \%)$ & 9.56 & 6.32 & $16.6(92 \%)$ \\
Mean SAR-2D (W/kg) & 12.8 & $0.38(97 \%)$ & 0.35 & 0.32 & $0.38(97 \%)$ & 0.34 & 0.28 & $0.36(97 \%)$ \\
\hline
\end{tabular}

Cost function: $\eta \times\left|M_{\mathrm{t}}\right|$-inhomogeneity $+(1-\eta) \times$ SAR-Max, Case I: $(0-80)+(80-150)+(150-500 \mathrm{~mm})$, Case II: $(0-200)+(200-500 \mathrm{~mm})$.

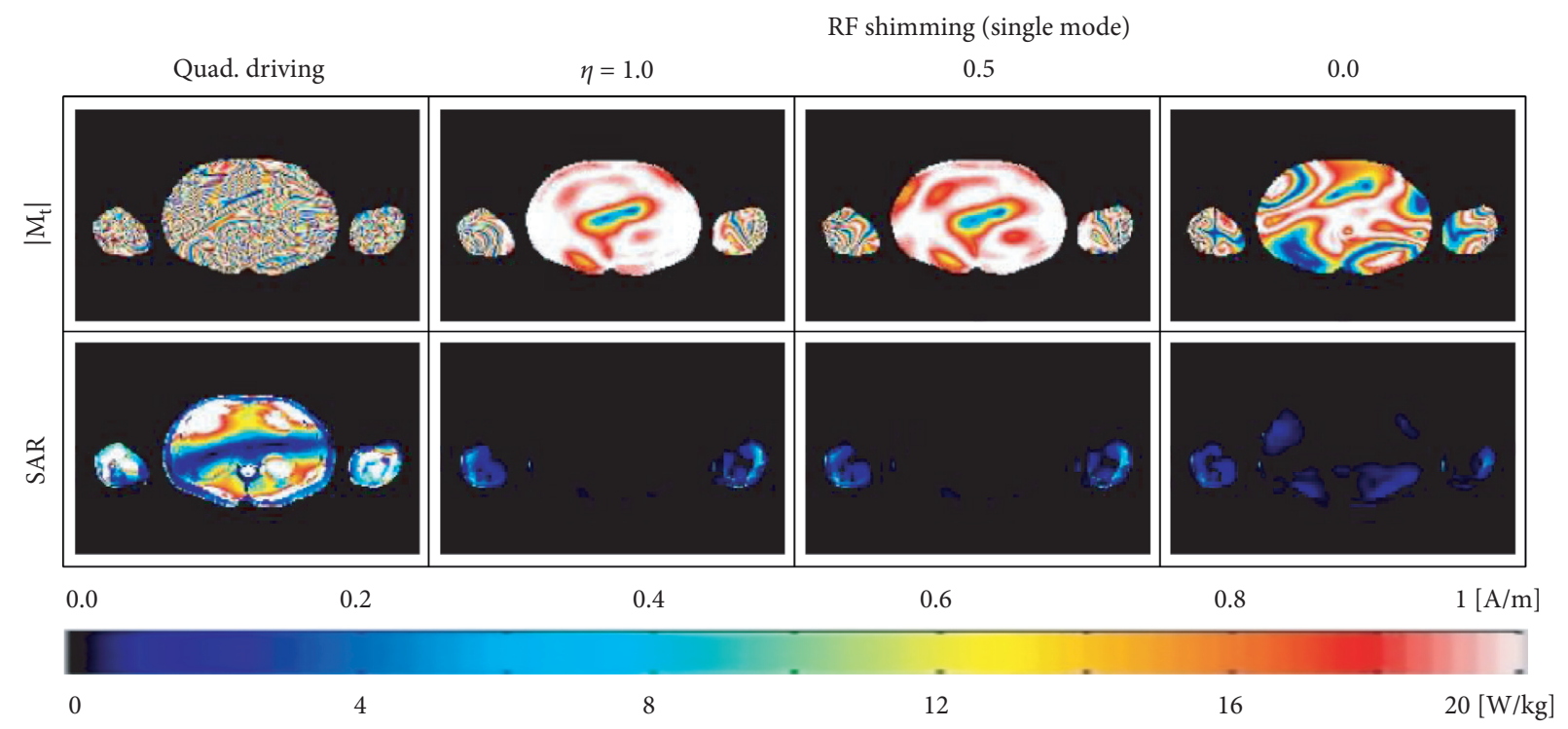

FiguRE 5: Numerical simulation results of $|\mathrm{Mt}|$ (first row) and corresponding unaveraged SAR (second row) using the human model (Ella) and two different optimization methods of quadrature driving and RF shimming with different cost function $(\eta)$ values, considering uniformity of $|\mathrm{Mt}|$ and SAR at $7.0 \mathrm{~T}$.

Figure 4 shows numerical simulation results of $2 \mathrm{D}-\left|M_{t}\right|$ acquired using the cylindrical phantom and four different optimization methods, i.e., quadrature driving, RF shimming using the single mode, RF shimming using the multimode, and RSMR method at 7.0 T. The corresponding mean and SD values of $\left|M_{t}\right|$ for each method are shown in Table 2. Compared to the results of quadrature driving method, mean and SD of $\left|M_{t}\right|$ were improved 39\% (mean) and 35\% (SD) using RF shimming with single mode without multiple ROIs, whereas 61\% (mean) and 94\% (SD) using the RSMR method. The uniformity of $\left|M_{t}\right|$ acquired using the RSMR method was improved especially in the peripheral region of the selected phantom image compared to that of other methods. The calculated unaveraged SAR values using the cylindrical phantom were not shown because of a small value (less than $1 \mathrm{~W} / \mathrm{kg}$ within the ID of $260 \mathrm{~mm}$ ).

Figures 5 and 6 show numerical simulation results of $\left|M_{t}\right|$ (first row) and corresponding unaveraged SAR (second row) acquired with different optimization methods of quadrature driving, single-mode RF shimming (Figure 5), and RSMR method (Figure 6) using the Ella model. The corresponding analytical results are shown in Table 3. Note that compared to the results of RF shimming using a single mode, the improvement in $\left|M_{t}\right|$ uniformity and SAR values was similar considering ROI of the ID of $500 \mathrm{~mm}$ (Table 3). However, the improvement was much better at the center region (Figures 5 and 6). Specifically, mean $\left|M_{t}\right|-2 \mathrm{D}=0.95[\mathrm{~A} / \mathrm{m}], \quad S D\left|M_{t}\right|-$ $2 \mathrm{D}=0.10, \quad$ MaxSAR $-2 \mathrm{D}=1.31 \quad[\mathrm{~W} / \mathrm{kg}]$, and mean SAR $-2 \mathrm{D}=0.11[\mathrm{~W} / \mathrm{kg}]$ within the region of $0-200 \mathrm{~mm}$ in case II $(\eta=1.0)$.

This is consistent with the results of the cylindrical phantom having a smaller ROI of $260 \mathrm{~mm}$ (Table 2). The improvement in $\left|\mathrm{M}_{\mathrm{t}}\right|$ uniformity was 35\% (RF shimming single-mode) and $52 \%$ (RF shimming multi-mode), whereas it was $94 \%$ at RSMR.

\section{Discussion}

The principle of RSMR is based on the premise that it is difficult to achieve adequate image homogeneity in regions larger than one wavelength in tissue; i.e., when the ID of ROIs is close to the wavelength, the $S D$ of $\left|M_{t}\right|$ was significantly increased with the RF shimming optimization method (Figure 3). Therefore, it is necessary to make multiple ROIs considering wavelength and electromagnetic properties of the tissue loading the coil. Then, the imaging parameters including RF coil design, amplitude, and phase 


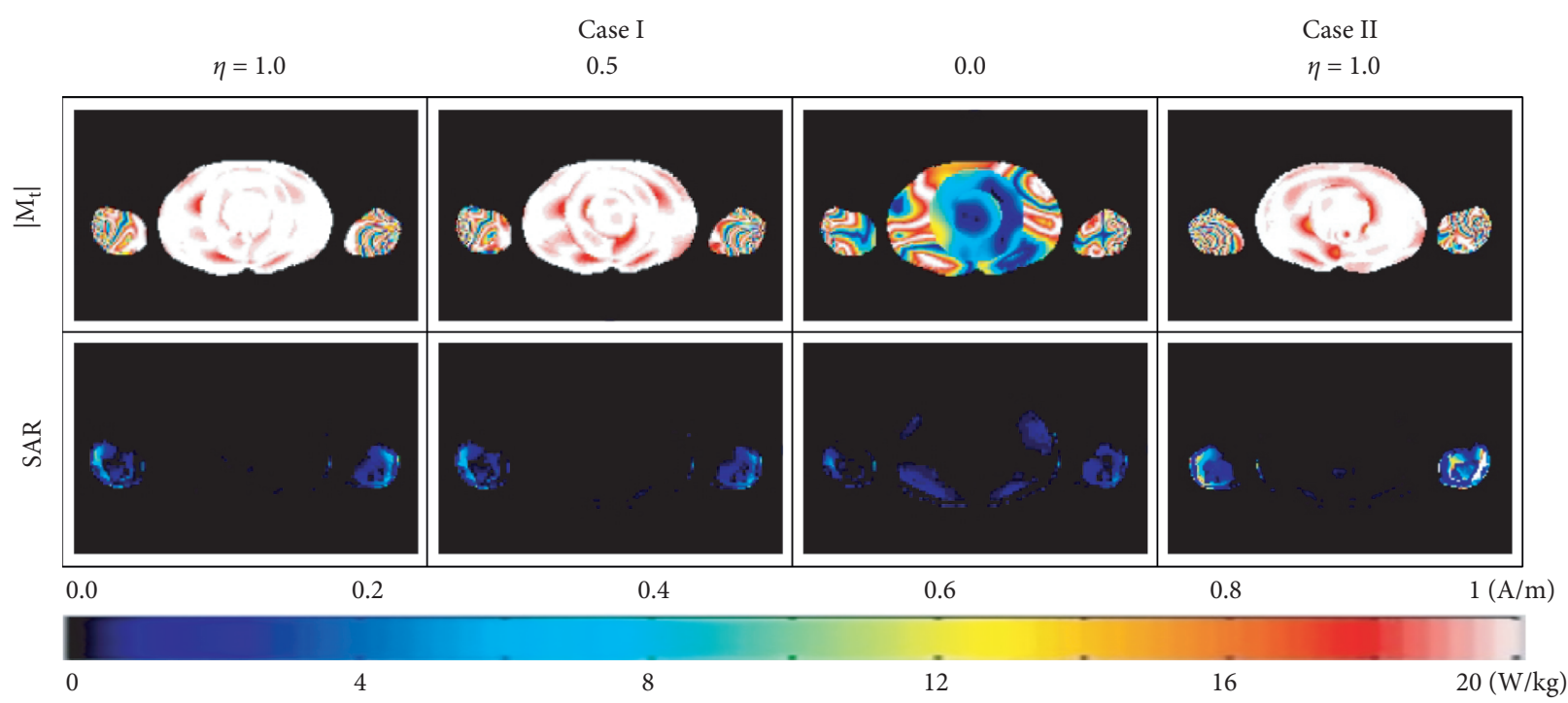

FIGURE 6: Numerical simulation results of $|\mathrm{Mt}|$ (first row) and corresponding unaveraged SAR (second row) using the Ella model and the RSMR optimization method with different cost function $(\eta)$ values and two different segmentation cases, i.e., Case I: $(0-80)+(80-150)+$ $(150-500 \mathrm{~mm})$; Case II: $(0-200)+(200-500 \mathrm{~mm})$ at $7.0 \mathrm{~T}$.

of each channel should be optimized. The image postprocessing within each ROI yields uniformly high $\left|M_{t}\right|$ and lower SAR distributions.

Compared to previous researches [7-11, 14-17, 24-26], the novelty of our designed method is using the independent multiple ROIs to optimize a big imaging region. Some previous research $[9,10]$ used multiple optimizations using different driving modes, which is similar to our method. Also, some improvements were shown compared to the results of quadrature driving and RF shimming with a single mode, which is consistent with the results in this study (Table 2 and Figure 4). However, they used one big ROI to cover the whole imaging region making a limitation to produce uniform $\left|M_{t}\right|$ and lower SAR distributions within the ROI having a size bigger than or close to one wavelength (Figures 4-6). It is because the current optimization methods including multi-mode RF shimming [10] have limitations to make uniform $\left|M_{t}\right|$ within a big ROI even though the initial mode of $B_{1}{ }^{+}$was different. In our method, the initial parameters were optimized at the specific ROIs which is smaller than the whole ROI, resulting in better $\left|M_{t}\right|$ uniformity and lower SAR, especially at the center region. This would provide enough image uniformity at the torso imaging.

One drawback of the designed RSMR method is the increased data acquisition time because of multiple data acquisitions and postprocessing compared to the standard MR imaging method. This increased acquisition time can be compensated using (1) parallel imaging methods, including SENSE [40], GRAPPA [41], and compressed sensing [42], and/or (2) decreased number of averages in the imaging parameters because of higher SNR at 7.0 T MRI compared to lower $\left|B_{0}\right|$ MR systems, e.g., $1.5 \mathrm{~T}$ and $3.0 \mathrm{~T}$.

The results shown in this study can be slightly changed depending on the selection of initial values of optimization parameters, e.g., magnitude and phase of each channel and increment parameter of magnitude and phase. Thus, a judicious choice of start values is needed. The proposed RSMR method can be applied to any arbitrary-shaped ROIs, e.g., heart, liver, and tumor.

The MRI experimental results using designed RSMR method and human body, e.g., Torso or CTL spine imaging, were not shown in this study because of nonavailability of the $7.0 \mathrm{~T}$ whole-body human MRI system.

\section{Conclusions}

This study shows that the designed RSMR method improved uniformity of $\left|\mathrm{M}_{\mathrm{t}}\right|$ and SAR within the cylindrical phantom and the human model compared to the results of quadrature driving and RF shimming with single mode and multi-mode using numerical simulations at 7.0 T. The designed method would be more efficient to the applications having bigger ROIs with a higher operating frequency. The methods and results presented here can provide useful information for improving uniformity and sensitivity of MR images in highfield human and animal MR imaging.

\section{Data Availability}

The data used to support the findings of this study are available on request through the corresponding author.

\section{Ethical Approval}

All procedures performed in studies were in accordance with the ethical standards of the institutional and/or national research committee and with the 1964 Helsinki Declaration and its later amendments or comparable ethical standards.

\section{Disclosure}

The mention of commercial products, their sources, or their use in connection with material reported herein is not 
to be construed as either an actual or implied endorsement of such products by the Department of Health and Human Services.

\section{Conflicts of Interest}

The authors declare that they have no conflicts of interest.

\section{References}

[1] D. I. Hoult, "The principle of reciprocity in signal strength calculations?A mathematical guide," Concepts in Magnetic Resonance, vol. 12, no. 4, pp. 173-187, 2000.

[2] J. T. Vaughan, M. Garwood, C. M. Collins et al., "7T vs. 4T: RF power, homogeneity, and signal-to-noise comparison in head images," Magnetic Resonance in Medicine, vol. 46, no. 1, pp. 24-30, 2001.

[3] C. M. Collins and M. B. Smith, "Signal-to-noise ratio and absorbed power as functions of main magnetic field strength, and definition of $90^{\circ} \mathrm{RF}$ pulse for the head in the birdcage coil," Magnetic Resonance in Medicine, vol. 45, no. 4, pp. 684-691, 2001.

[4] C. M. Collins and M. B. Smith, "Calculations ofB1 distribution, SNR, and SAR for a surface coil adjacent to an anatomically-accurate human body model," Magnetic Resonance in Medicine, vol. 45, no. 4, pp. 692-699, 2001.

[5] B. S. Park, B. McCright, L. M. Angelone, A. Razjouyan, and S. S. Rajan, "Improvement of electromagnetic field distributions using high dielectric constant (HDC) materials for CTLspine MRI: numerical simulations and experiments," IEEE Transactions on Electromagnetic Compatibility, vol. 59, no. 5, pp. 1382-1389, 2017.

[6] A. G. Webb and C. M. Collins, "Parallel transmit and receive technology in high-field magnetic resonance neuroimaging," International Journal of Imaging Systems and Technology, vol. 20, no. 1, pp. 2-13, 2010.

[7] B. S. Park, S. S. Rajan, J. W. Guag, and L. M. Angelone, "A novel method to decrease electric field and SAR using an external high dielectric sleeve at 3 T head MRI: numerical and experimental results," IEEE Transactions on Biomedical Engineering, vol. 62, no. 4, pp. 1063-1069, 2015.

[8] N. I. Avdievich, S. Oh, H. P. Hetherington, and C. M. Collins, "Improved homogeneity of the transmit field by simultaneous transmission with phased array and volume coil," Journal of Magnetic Resonance Imaging, vol. 32, no. 2, pp. 476-481, 2010.

[9] V. Taracila, L. S. Petropoulos, T. P. Eagan, and R. W. BROWN, "Image uniformity improvement for birdcage-like volume coils at $400 \mathrm{MHz}$ using multichannel excitations," Concepts in Magnetic Resonance Part B: Magnetic Resonance Engineering, vol. 29B, no. 3, pp. 153-160, 2006.

[10] S. Orzada, S. Maderwald, B. A. Poser, A. K. Bitz, H. H. Quick, and M. E. Ladd, "RF excitation using time interleaved acquisition of modes (TIAMO) to address B 1 inhomogeneity in high-field MRI," Magnetic Resonance in Medicine, vol. 64, no. 2, pp. 327-333, 2010.

[11] C. M. Collins, W. Liu, B. J. Swift, and M. B. Smith, "Combination of optimized transmit arrays and some receive array reconstruction methods can yield homogeneous images at very high frequencies," Magnetic Resonance in Medicine, vol. 54, no. 6, pp. 1327-1332, 2005.

[12] N. Maril, C. M. Collins, R. L. Greenman, and R. E. Lenkinski, "Strategies for shimming the breast," Magnetic Resonance in Medicine, vol. 54, no. 5, pp. 1139-1145, 2005.
[13] P.-F. Moortele et al., "B1 destructive interferences and spatial phase patterns at $7 \mathrm{~T}$ with a head transceiver array coil," Magnetic Resonance in Medicine, vol. 54, pp. 1503-1518, 2005.

[14] W. Mao, M. B. Smith, and C. M. Collins, "Exploring the limits of RF shimming for high-field MRI of the human head," Magnetic Resonance in Medicine, vol. 56, no. 4, pp. 918-922, 2006.

[15] Z. Cao, J. Park, Z.-H. Cho, and C. M. Collins, "Numerical evaluation of image homogeneity, signal-to-noise ratio, and specific absorption rate for human brain imaging at 1.5, 3, 7, 10.5 , and $14 \mathrm{~T}$ in an 8-channel transmit/receive array," Journal of Magnetic Resonance Imaging, vol. 41, no. 5, pp. 1432-1439, 2015.

[16] C. M. Collins, Z. Wang, W. Mao, J. Fang, W. Liu, and M. B. Smith, "Array-optimized composite pulse for excellent whole-brain homogeneity in high-field MRI," Magnetic Resonance in Medicine, vol. 57, no. 3, pp. 470-474, 2007.

[17] B. Park, K. Sung, J. McGarrity et al., "Slice-selective transmit array pulses for improvement in excitation uniformity and reduction of SAR," Journal of Electromagnetic Analysis and Applications, vol. 5, no. 05, pp. 205-212, 2013.

[18] K. Setsompop, L. L. Wald, V. Alagappan et al., "Parallel RF transmission with eight channels at 3 tesla," Magnetic Resonance in Medicine, vol. 56, no. 5, pp. 1163-1171, 2006.

[19] C. Ma, D. Xu, K. F. King, and Z.-P. Liang, "Joint design of spoke trajectories and RF pulses for parallel excitation," Magnetic Resonance in Medicine, vol. 65, no. 4, pp. 973-985, 2011.

[20] H. Sun, "Spoke pulse design IN magnetic resonance imaging using greedy minimax algorithm," in Proceedings of the IEEE 10th International Symposium on Biomedical Imaging: From Nano to Macro, pp. 696-699, San Francisco, CA, USA, April 2013.

[21] M. A. Ert€urk, A. J. E. Raaijmakers, G. Adriany, K. Ugurbil, and G. J. Metzger, "A 16-channel combined loop-dipole transceiver array for 7 tesla body MRI," Magnetic Resonance in Medicine, vol. 77, pp. 884-894, 2017.

[22] M. A. Ert€urk et al., "Toward imaging the body at 10.5 tesla," Magnetic Resonance in Medicine, vol. 77, pp. 434-443, 2017.

[23] L. Alon, R. Lattanzi, K. Lakshmanan et al., "Transverse slot antennas for high field MRI," Magnetic Resonance in Medicine, vol. 80, no. 3, pp. 1233-1242, 2018.

[24] G. Chen, C. M. Collins, D. K. Sodickson, and G. C. Wiggins, "A method to assess the loss of a dipole antenna for ultrahigh-field MRI," Magnetic Resonance in Medicine, vol. 79, no. 3, pp. 1773-1780, 2018.

[25] Q. X. Yang, J. Wang, J. Wang, C. M. Collins, C. Wang, and M. B. Smith, "Reducing SAR and enhancing cerebral signalto-noise ratio with high permittivity padding at $3 \mathrm{~T}$," Magnetic Resonance in Medicine, vol. 65, no. 2, pp. 358-362, 2011.

[26] Q. X. Yang, S. Rupprecht, W. Luo et al., "Radiofrequency field enhancement with high dielectric constant (HDC) pads in a receive array coil at 3.0T," Journal of Magnetic Resonance Imaging, vol. 38, no. 2, pp. 435-440, 2013.

[27] W. Luo, M. T. Lanagan, C. T. Sica et al., "Permittivity and performance of dielectric pads with sintered ceramic beads in MRI: early experiments and simulations at 3 T," Magnetic Resonance in Medicine, vol. 70, no. 1, pp. 269-275, 2013.

[28] M. V. Vaidya, C. M. Deniz, C. M. Collins, D. K. Sodickson, and R. Lattanzi, "Manipulating transmit and receive sensitivities of radiofrequency surface coils using shielded and unshielded high-permittivity materials," Magnetic Resonance Materials in Physics, Biology and Medicine, vol. 31, no. 3, pp. 355-366, 2018. 
[29] G. J. Metzger, C. Snyder, C. Akgun, T. Vaughan, K. Ugurbil, and P.-F. Van de Moortele, "LocalB1+ shimming for prostate imaging with transceiver arrays at 7T based on subject-dependent transmit phase measurements," Magnetic Resonance in Medicine, vol. 59, no. 2, pp. 396-409, 2008.

[30] X. Wu, S. Schmitter, E. J. Auerbach, K. Uğurbil, and P.-F. V. D. Moortele, "Mitigating transmit B 1 inhomogeneity in the liver at 7T using multi-spoke parallel transmit RF pulse design," Quantitative Imaging in Medicine and Surgery, vol. 4, pp. 4-10, 2014.

[31] B. K. Li, F. Liu, and S. Crozier, "High-field magnetic resonance imaging with reduced field/tissue RF artefacts-A modeling study using hybrid MoM/FEM and FDTD technique," IEEE Transactions on Electromagnetic Compatibility, vol. 48, no. 4, pp. 628-633, 2006.

[32] F. Liu, B. L. Beck, J. R. Fitzsimmons, S. J. Blackband, and S. Crozier, "A theoretical comparison of two optimization methods for radiofrequency drive schemes in high frequency MRI resonators," Physics in Medicine and Biology, vol. 50, no. 22, pp. 5281-5291, 2005.

[33] The Federal Communications Commission, Body Tissue Dielectric Parameters, The Federal Communications Commission, Washington, DC, USA, 1996, https://www.fcc.gov/ general/body-tissue-dielectric-parameters (accessed.

[34] C. Gabriel, "Compilation of the dielectric properties of body tissues at RF and microwave frequencies," Air Force Materiel Command, Brooks Air Force Base, Texas, TX, USA, AL/OETR-1996-0037, 1996.

[35] J. Hamblin, This is the average man's body, The Atlantic, Washington, DC, USA, 2013, https://www.theatlantic. com/health/archive/2013/10/this-is-the-average-mans-body/ 280194/ (accessed.

[36] Z. Cao, S. Oh, C. T. Sica et al., "Bloch-based MRI system simulator considering realistic electromagnetic fields for calculation of signal, noise, and specific absorption rate," Magnetic Resonance in Medicine, vol. 72, no. 1, pp. 237-247, 2014.

[37] A. Christ, W. Kainz, E. G. Hahn et al., "The Virtual Familydevelopment of surface-based anatomical models of two adults and two children for dosimetric simulations," Physics in Medicine and Biology, vol. 55, no. 2, pp. N23-N38, 2010.

[38] C. Gabriel, T. Y. A. Chan, and E. H. Grant, "Admittance models for open ended coaxial probes and their place in dielectric spectroscopy," Physics in Medicine and Biology, vol. 39, no. 12, pp. 2183-2200, 1994.

[39] S. Gabriel, R. W. Lau, and C. Gabriel, "The dielectric properties of biological tissues: II. Measurements in the frequency range $10 \mathrm{~Hz}$ to $20 \mathrm{GHz}$," Physics in Medicine and Biology, vol. 41, no. 11, pp. 2251-2269, 1996.

[40] K. P. Pruessmann, M. Weiger, M. B. Scheidegger, and P. Boesiger, "SENSE: sensitivity encoding for fast MRI," Magnetic Resonance in Medicine, vol. 42, no. 5, pp. 952-962, 1999.

[41] M. A. Griswold, P. M. Jakob, R. M. Heidemann et al., "Generalized autocalibrating partially parallel acquisitions (GRAPPA)," Magnetic Resonance in Medicine, vol. 47, no. 6, pp. 1202-1210, 2002.

[42] D. L. Donoho, "Compressed sensing," IEEE Transactions on Information Theory, vol. 52, no. 4, pp. 1289-1306, 2006. 